These two cases, both in mares, one four, the other nine years old, certainly encourage operative interference. Although the older animal required to be operated on twice, and evidently considerable violence used to effect the removal of the offending substance, a favourable recovery is recorded. But there can be nothing more certain than that in the two cases related in this paper operative interference would have had little, if any, chance of being successful.

\title{
EXPERIMENTAL DEMONSTRATION OF THE UNITY OF TUBERCULOSIS ${ }^{1}$
}

\author{
By S. Arloing, Lyons.
}

TEN years ago there seemed to be general agreement with regard to the unity of tuberculosis in mammals. A few pathologists, in imitation of Virchow, perhaps maintained some idea of keeping separate bovine tuberculosis, the lesions of which appeared to them to be of a special nature. But their tendencies were repressed by the fact that pathogenic properties were similar in human and bovine tuberculosis.

Moreover, it is at least tacitly in favour of this opinion, which is based principally on the presence of Koch's bacilli in all the lesions, and on the experimental production of tuberculosis with infecting products either of human or bovine origin, that steps were everywhere taken to regulate the use of animal products suspected of containing the tuberculous virus.

Doubts with regard to an absolute identity were introduced in 1892 , with the works of Theobald Smith, R. Gaiser, Frothingham, and Dinwiddie.

Th. Smith, who first took up this question, and Dinwiddie, made the most extensive comparative studies of human and bovine tuberculosis. Smith had been struck by the morphological differences offered by the two bacilli. According to him, the bacilli of bovine origin were short, straight, thick, and of uniform diameter, sometimes swollen in the middle, and strongly resisted decoloration by acids. The bacilli of human origin were thinner, longer, more or less curved, grouped in larger or smaller numbers, and less resistant to decoloration by acids. On solid serum, the culture of the bovine bacillus spread evenly over the nutritive medium, and assumed the appearance of a scarcely visible veil; while that of the human bacillus yielded warty masses, which were dry on the surface.

After some experiments on inoculation, Smith also pointed out some differences in respect of pathogenic properties. The bovine bacillus generally manifested a greater virulence for animals than the human bacillus, a difference observed by Villemin. In spite of this, he concluded, not that there were two distinct species, but that the characters of human and bovine bacilli were variable. According to him, the tubercle bacilli of mammals form a compact group, in which, nevertheless, varieties exist.

Dinwiddie's first work, dated I 899, concerned the question of the relative virulence of human and bovine tuberculosis for the domestic

1 Translated from the "Journal de Méd. Vétérinaire," May 1903. 
animals. He had been inspired by the researches of Smith and Frothingham. His other works are dated IgOo-Igor.

Dinwiddie found that infective material of bovine origin is more active than that of human origin, that the bacilli present analogous differences, and that bovine tuberculous matter presents a variable activity. He estimates that the lesions peculiar to tuberculosis of the ox in its typical form are due to a particular kind of reaction on the part of the animal, rather than to any special characters which would distinguish bovine from human bacilli. $\mathrm{He}$ thinks that the danger of man being infected by cattle has been generally exaggerated. Nevertheless, as the differences which he has observed denote degrees of virulence, rather than different virulences, he thinks it always wise to take measures of precaution, especially with regard to milk.

Frothingham and Gaiser made fewer experiments, from which it appeared that human tuberculosis was unable to propagate in the calf further than at the point of inoculation. But, we repeat, not one of the previous authors dreamt of making two distinct specific groups of the two forms of tuberculosis. On the contrary, this idea occurred to Professor Robert Koch, and he expressed it, under solemn circumstances, at a general meeting of the British Congress on Tuberculosis, in July IgoI. In the experiments which led him to this subversive opinion, Koch had for co-worker Professor Schuitz, of the Berlin Veterinary School. In support of their proposition, Koch and Schuitz have presented arguments of two kinds: some, borrowed from clinical observation, have simply the value of a probability; the others, drawn from experiments, are, on the contrary, of great value, as they can be submitted to a rigorous control.

In this work we will only take up the last named. We will examine the former in a later study.

\section{THE EXPERIMENTAL CRITERION OF KOCH AND SCHÜTZ.}

On the ground of thirty-four very expensive experiments, in which the inoculation of human and bovine tuberculosis in large herbivorous or omnivorous animals was comparatively studied, Koch and Schütz affirm that these two forms of tuberculosis are different, because the human bacilli, contrary to the bacilli of bovine origin, do not infect cattle, whatever the means of introduction may be.

By cattle, these savants mean the ox, sheep, pig, goat, and donkey.

Koch and Schuitz call to their aid the results obtained by some previous experimenters, notably Chauveau, Gunther and Harms, Bollinger, Smith, Dinwiddie, and Frothingham.

We have already said that the three last-named authors. had studied the comparative inoculation of the bacilli of the two forms of tuberculosis, as well as the evolution and characteristics of the two kinds of bacilli; that they had pointed out differences, and that they had even raised the question of separation before the experimenters of Berlin, but had stopped en route.

Koch and Schiitz had no such hesitation; but, in affirming that Chauveau, Gunther, and Harms arrived at the conclusions which they expressed in London, they are mistaken. I will not describe the 
experiments of M. Chauveau. It will be enough to recall that this experimenter obtained the infection of the ox by feeding with products of human and bovine tuberculosis under its different forms. The conclusions which he drew from this were so clear that, in his opening address at the Congress for the study of tuberculosis, held at Paris in I888, he did not hesitate to write: "Therefore nothing is lacking in the demonstration of the identity, originally not recognised, of the two forms of tuberculosis. There is only one disease, only one virus, attacking both the bovine and the human species, and capable of being transmitted from the one to the other."

Bollinger, on his side, after numerous experiments made by himself or his pupils, chiefly on the pig, expressed himself several times in favour of identity, notably in 1899 , at the Berlin Congress. On this occasion he proclaimed that tuberculosis of cattle and pigs is, from the point of view of etiology, identical with that of man; because of its enormous extension, it is not only a veritable scourge of breeding and agriculture, but also a serious danger for man.

As for Gunther and Harms, they had no particular reasons for figuring in the question raised by Koch and Schuitz, for in the interesting experiments that they performed on the contagion of tuberculosis they never took the infecting material from man. This was taken from the ox, save in one case, where it was taken from the monkey.

It will then be seen that Koch and Schütz have not been very happy in the choice of their references. Hence, on them, and on them alone, rests the responsibility for the dualistic theory, by the aid of which they intended to revolutionise the prophylaxis of tuberculosis.

From these few lines we conclude that Koch's opinion rests on the non-inoculability of human tuberculosis to herbivorous animals or the pig. Let us see, by the help of our own experiments, whether this text is unimpeachable, and whether it can serve as the foundation of a new idea.

\section{THE AUTHOR'S OWN EXPERIMENTS REGARDING THE}

INOCULABILITY OF HUMAN TUBERCULOSIS TO LARGE ANIMALS.

We have successfully inoculated the bacillus of human tuberculosis to the donkey, the goat, the sheep, the ox, and the pig. Let us briefly describe these inoculations.

(a) Inoculation of Human Tuberculosis to the Ass.-The experiments on this point go back to 1896 ,

Some particles were taken from the surface of a culture of human bacilli on glycerinised potato, and made into an emulsion with water. These bacilli had been isolated from the lesions in a guinea-pig; that is to say, they infected this animal very easily.

With the emulsion, previously filtered through sterilised linen, two donkeys were inoculated in the jugular vein. One of the donkeys, which was not very strong, rapidly became weak, and died at the end of twenty-eight days.

The other, young and vigorous, continued to appear healthy. However, the normal temperature oscillated in the course of the first 
month, and rose sometimes to $I^{\circ}$ above normal. It was killed at the end of two months.

The post-morten revealed tuberculous lesions in the lungs of both subjects. In the first, congestive lesions were found here and there, and everywhere little tubercles which felt firm to the finger. In the second, in passing the finger over the surface of the lungs, one felt a large number of circumscribed points where the consistence of the tissue was increased. These corresponded to tuberculous nodules in a state of retrogression, as was proved by the histological examination. The miscroscope has likewise shown that the nodules in the lungs of the first donkey were tubercles in process of formation.

In another experiment two intravenous inoculations were made at intervals of a fortnight. The temperature oscillated after each injection. The maximum was $1^{\circ}$ in the first period, and $1.5^{\circ}$ in the second. When tested with tuberculin after the second injection, the donkey had an elevation of temperature of $2.9^{\circ} \mathrm{C}$. It was killed two months from the beginning of the experiment.

The post-mortem showed a great number of little elevations in the lungs, often surrounded by a little ring of congestion; and a great number of round whitish spots of the diameter of a lentil. The histological examination showed that the bright red points were tuberculous granulations, isolated or close together, and in full activity; while the whitish spots corresponded with tuberculous granulations of which the cellular elements were more or less compressed by the connective-tissue fibrils.

Hence, certain pure cultures of human bacilli are capable of infecting the donkey when they are introduced into a vein. Hence, also, the donkey can recover spontaneously from pulmonary tuberculosis produced by an intravenous injection of human bacilli, a remark already made by M. Chauveau. ${ }^{1}$

(b) Inoculation of Goats with. Human Tuberculosis.-The experiments described in this paragraph were commenced 2oth April 1899. At this date, seven goats received into the jugular vein an injection of an emulsion of Koch's bacilli of human origin, taken from a culture on glycerinised potato. Three goats had not previously been the subject of any experiment. Four had been treated for a long time with substances which have a certain reputation in the therapeutics of tuberculosis. Two of the goats previously untreated succumbed in less than a month. The five others were killed on the 8 th July of the same year. The goats which died quickly grew thin, coughed, and experienced difficulty in respiration almost immediately; their temperature was frequently above $40^{\circ} \mathrm{C}$, and even went up to $4 \mathrm{I}^{\circ} 3^{\circ} \mathrm{C}$.

The post-mortem showed considerable lesions in the lungs, all consisting of tuberculous granulations, as was seen from the histological examination.

All the others showed signs of infection in different degree-elevation of temperature a few days after the injection, cough after the eighth or tivelfth day, and emaciation. During the last month their temperature oscillated between $39^{\circ} \mathrm{I}^{\circ}$ and $39^{\circ} 7^{\circ} \mathrm{C}$. At the post-mortem the lungs presented a tuberculous eruption. This eruption was composed of

1 These experiments were published in the Journal de Physiologic et de Pathologie générale, for July 1900. 
granulations of which the largest were of the diameter of a millet-seed. The granulations were confluent on the anterior lobes of the lungs. The lymphatic glands at the entrance to the chest, and those in the mediastinum near the oesophagus, were more or less swollen. Some of the granulations showed commencing caseous degeneration towards their centres. ${ }^{1}$

(c) Inoculation of Human Tuberculosis to the Ox, Sheep, and Goat.In this paragraph five series of experiments made after the communication of Koch and Schütz in London will be described.

Each series comprised one or two young bovine animals, one or two sheep, and one or two goats. All these animals were recognisect as being free from tuberculosis. Tuberculin was used according to directions in the case of the bovine animals.

In addition to the proper subjects of each series, we used a few rabbits and guinea-pigs in order-to obtain information with regard to the virulence of the infecting material used in each experiment. This infecting material consisted of human bacilli cultivated on glycerinised potato. For each series human bacilli taken from different patients were used, so that our experiments are not the repetition of a simple test; in this respect they differ from those of Koch and some other experimenters. We used different bacilli because we knew the extent to which tubercle bacilli can undergo modifications from the pathogenic point of view.

The bacilli were made into an emulsion with water containing 7 per cent. of common salt. The emulsion was made in fixed proportions, generally one part of bacilli to twenty-five of water, then filtered through a fine linen sieve, and finally injected into the jugular vein or an auricular vein. The intravenous injection has been almost uniformly adopted so as to render comparison of experiments easier, and infection more certain.

First Series.-Emulsion of $\mathrm{I}$ in 25 of a human bacillus isolated from the sputum of a patient, and cultivated on potato; of medium activity for the rabbit and guinea-pig. With this the following injections were made :-

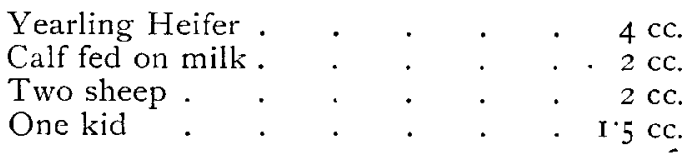

One sheep and the kid died tuberculous in thirty-seven days. The calf died accidentally at the end of thirty-two days, but after having wasted away. The other sheep and the heifer were killed, the former at the end of twenty-five days, the latter after I 20 days. Discrete tubercles in the lungs of the heifer; exquisite eruption of tuberculous granulations in the calf and the sheep; more diffuse and extensive lesions in the kid.

Otherwise expressed, complete success in all subjects; moreover, verified by histological examination.

Second Series.-Emulsion of I in 25 of bacilli obtained from a case of tuberculous pleurisy, which subsequently recovered. These bacilli were passed twice through the guinea-pig and afterwards

1 These experiments on the goat and ass enabled me, in the next issue of the Rerue de la Tuberculose (August 1901), to dissent from the view expressed by Koch in London. 
cultivated on glycerinised potato and solid blood. The following were inoculated with the emulsion :-

$$
\begin{aligned}
& \text { One weakly calf . . . . . } 2 \text { cc. } \\
& \text { Two sheep each . . . . . I I } 5 \mathrm{cc} \text {. } \\
& \text { One goat . . . . . } \mathrm{I}_{5} 5 \mathrm{cc} \text {. }
\end{aligned}
$$

The calf died of tuberculosis at the end of seventeen days; the sheep and the goat recovered after having presented signs of infection, and were killed eighty days after inoculation.

At the post-mortem a great number of nascent tubercles were found in the calf; in the other subjects a tuberculous infiltration of the lungs and of the pulmonary pleura, not so considerable, however, in the goat as in the sheep.

Microscopic examination showed that the liver and spleen of the sheep and goat were affected with tuberculosis.

Third Series.-Bacilli obtained from a fatal case of tuberculosis involving the pleura, pericardium, and lungs; cultivated in the same manner as those in the preceding series. With the $I$ in 25 emulsion the following were inoculated :-

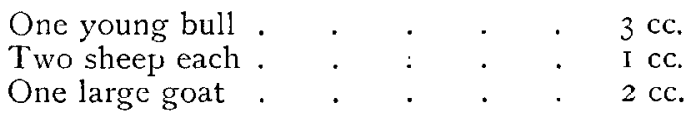

The bull, previously healthy, died of tuberculosis in thirty-two days, having shown frightful difficulty in respiration. One sheep died accidentally. The other sheep and the goat were killed forty-two days after inoculation.

In the young bull the lungs were crammed with fine tubercles surrounded by inflammation; the bronchial and mediastinal glands were enormous. Liver yellowish and fatty in appearance.

A great number of small sub-pleural and intra-parenchymatous tubercles in the sheeps' lungs; smaller in the goat. The microscope revealed a generalised fatty degeneration of the liver, and a great number of small young tubercles, often situated within the lobules.

Fourth Series.-Emulsion, I in 25, of bacilli obtained from the sputum of a tuberculous patient, and cultivated in a state of purity on glycerinised potato. With this the following were inoculated :-

$$
\begin{aligned}
& \text { One heifer . . . . . } 5 \text { cc. } \\
& \text { One sheep . . . . . } 2 \text { cc, } \\
& \text { One sheep . . . . . } 2 \mathrm{cc} \text {, in the peritoneum. }
\end{aligned}
$$

The emulsion was diluted to $\mathbf{I}: 500$, and then used to inject into the jugular of

$$
\text { One calf. . . . . } 2 \mathrm{cc} \text {. }
$$

The calves, except for a slight cough, appeared to be healthy; however tuberculin caused a marked reaction. They were killed at the end of I 80 days. The sheep also appeared healthy, but they reacted to tuberculin. They were killed ninety days after inoculation.

The lungs of the heifer appeared to be healthy; those of the sheep led one to suspect the presence of very minute sub-pleural tubercles. That is to say, a macroscopic examination seemed to indicate a complete failure. But with the microscope very fine tubercles could be 
seen in the pulmonary parenchyma, principally along the bronchial tubes. Failure was therefore only apparent, and the bacilli used for inoculation were not inactive. Moreover, the sheep inoculated in the peritoneum proves this, for in this animal the glands scattered in the furrows of the stomach were found to be hypertrophied and tuberculous. Their centre was transformed into a caseous mass, which had assumed the aspect of analogous lesions in bovine tuberculosis.

Fifth Series.-Emulsion, I in 25, prepared with cultures of a bacillus found in the glands of a man affected with dropsy. With this emulsion the following were inoculated in the jugular-

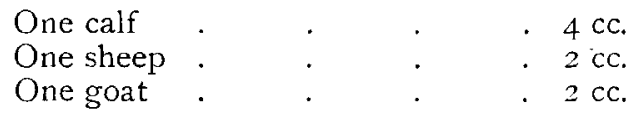

These animals grew slightly thinner during the fortnight following inoculation, and then gradually regained their former condition. However, they all reacted distinctly to tuberculin.

An inoculation similar to the first was made. The animals resisted admirably. They were killed between the I 20th and I 50 th day from the commencement of the experiment.

At the post-mortem the lungs appeared healthy. Very careful observations had to be made to find signs of subpleural lesions. However, the calf showed hypertrophy of several lymphatic glands. A pulp made from these glands caused tuberculosis in guinea-pigs. The hypertrophy was therefore of a tuberculous nature.

But, although the lungs appeared healthy to the naked eye, they contained fine lesions which were visible with the microscope. Nodular infiltrations were found in the liver and kidneys.

The five series of experiments described above may be divided into two groups. The first group includes the inoculations of the first, second, and third series. The three strains of human bacilli used in these experiments gave results which were undoubtedly positive. They caused pulmonary lesions which were numerous and obvious; moreover, microscopic tuberculous lesions were present in the liver, spleen, and kidneys, bearing witness to the generalisation of the tuberculous process.

The second group includes the fourth and fifth series. The bacilli used in these experiments only caused very slight lesions, which might have passed undetected in a superficial examination or by any person desiring a negative result. But the tuberculous lesions were manifest in the microscopic sections of the lung.

Bacilli of human origin can therefore infect the ox, sheep, and goat. However, it must be added that their virulence has presented various degrees.

(d) Inoculation of a Pig with Human Tuberculosis.-An emulsion of $I$ in 25 was made with the bacilli used for inoculation in the first series of experiments just described, and $4 \mathrm{cc}$. of this emulsion were injected into the auricular vein of three young pigs.

From the following day the three subjects appegared to be ill. Two remained lying down, and refused all food. Their condition became worse during the following days. A month and a half after inoculation it seemed that one of the pigs must shortly die, and it was 
slaughtered. On the contrary, the condition of the other two pigs improved; they were kept for thirteen months, growing and fattening like perfectly healthy subjects. They were killed.

The pig killed at the end of a month and a half presented some patches of pleurisy. At this level the pleura presented some large more or less projecting tubercles.

The bronchial and mediastinal glands were voluminous and tuberculous. The malpighian bodies of the spleen were hypertrophied, and some were tubercular.

The microscope confirmed all these lesions, and, further, allowed tuberculous alterations to be observed in the spleen, the liver, and the kidneys.

One of the subjects kept for thirteen months, fat and in good condition, presented here and there in the lungs tuberculous masses which were caseous in the centre, and varied between the size of a lentil and that of a haricot bean. The bronchial, mediastinal, and retro-pharyngeal glands, and a few mesenteric glands, were hypertrophied, and contained caseous tubercles. In the liver a few caseous nodules were found; in the spleen one was found near one of the extremities. The other pig, in very good condition, appeared at the first glance to have perfectly healthy lungs; but after slight dessication a few very small, whitish, pleural granulations were perceived. The bronchial, mediastinal, retro-pharyngeal, and mesenteric glands were tumefied and hard, and showed on section some sclerotic ponits, like old tubercles in the fibroid state.

To sum up, in all the three pigs the success of the experiment was complete; but in course of time the tuberculous process became circumscribed, and, according to the subject, the lesions underwent either caseous degeneration or fibroid transformation. The results in these experiments have been influenced by the predisposition or resistance peculiar to each subject.

(e) Inoculation of the Ox, Sheep, and Goat with Bovine Tuberculosis.It seemed indispensable that we too should compare the human bacilli with those of the ox in the matter of virulence. To this end we carried out three series of experiments similar to those described in paragraph $c$.

First Series.-Bacilli obtained from a tuberculous lesion in an ox, and grown as a pure culture on glycerinised potato. An emulsion of $I$ in 25 was injected into the jugular vein of-

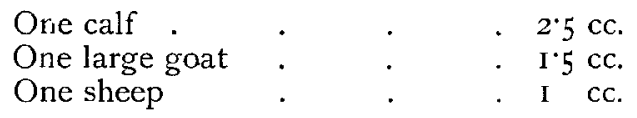

The calf very quickly became ill, presenting signs of very grave acute pulmonary tuberculosis; it died twenty-five days after inoculation. The sheep followcd suit, and died on the forty-fourth day. As for the goat, after having also shown alarming symptoms, it partially recovered. It was killed at the end of 150 days, being then in a rather sorry condition.

The lungs. of the calf were everywhere infiltrated with tuberculous granulations surrounded by inflammatory lesions. Enormous bronchial and mediastinal glands, recalling those of the calf in series 3 of inoculations with human tuberculosis. The retro-pharyngeal glands 
were hypertrophied, but without apparent tuberculous lesions; nevertheless, they contained bacilli, as was proved by the inoculation of a guinea-pig with pulp from them.

The lungs of the sheep were greatly distended by a generalised infiltration, in which the eye could easily discern a great number of tubercles. The spleen was hypertrophied, the follicles being more distinctly visible than usual. Some very fine granulations had formed in the liver.

The lungs of the goat were dotted, both under the pleura and in the depth of the parenchyma, with large grey tubercles without any peripheral inflammatory zone; some parts of the anterior lobes were hepatised. Many tuberculous granulations in the liver. Very few nodules in the spleen. The histological examination confirmed the results of the post-mortem.

Second Series.-Another bacillus of bovine origin, cultivated on glycerinised potato; emulsion, $I$ in 25 , inoculated into the following animals :-

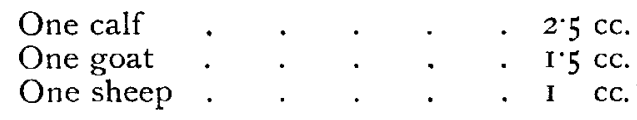

From the fourth day the calf began to cough; from the tenth the three animals suffered greatly-cough, rapid breathing, and fever.

The sheep died at the end of twelve days, the goat at the end of seventeen days, and the calf at the end of eighteen days.

Confluent eruption of fine tubercles in the lungs of the calf, causing here and there complete hepatisation. Bronchial and mediastinal glands hypertrophied and pulpy. In the sheep and the goat the pulmonary lesions were similar to those described in the calf. The sheep was more gravely attacked than the goat.

A second calf was inoculated with the same bacilli, only the emulsion was $I$ in 200 instead of $I$ in 25 . Little change was remarked in the animal during ten days, but suddenly it became very ill; it was killed when in a dying condition on the fiftieth day.

Tubercles were scattered in the lungs, larger but less confluent than those in the lungs of the calf which died soon after inoculation. In some parts of the right lung the tubercles were surrounded by recently-formed inflammatory lesions, rapidly approaching hepatisation.

Hence, a very weak dose of these bacilli can bring about fatal results.

Third Series.-In this series, the infecting material was taken from pleural tubercles in an ox, made into a pulp, diluted, and filtered. The following inoculations were made with it :-

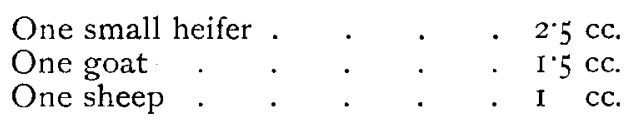

The heifer showed characteristic symptoms, and died twenty days after inoculation. The goat became rapidly ill, and died on the twenty-second day. The sheep died on the thirty-first day.

The lungs of the heifer were riddled with tubercles embedded in 
inflammatory lesions, which at some places rendered the tissue solid; the parenchymatous organs were congested and swollen, but not apparently tuberculous.

In the goat, the peri-tuberculous inflammation gave to the lungs an appearance of a greyish block, the fragments of which have fallen to the bottom of the water. The liver presented small tuberculous lesions. The lesions were almost identical in the sheep.

The matter taken from pleural tuberculous "grapes" of the ox thus acted in the same way as the pure culture of bovine bacilli used in the two preceding series.

$(f)$. Inoculation in the ox, sheep, and goat, with equine tuberculosis.In order to complete our comparative study, we made a series of inoculations with bacilli of equine origin cultivated on glycerinised potato. With an emulsion of $I$ in 25 we inoculated into the jugular :-

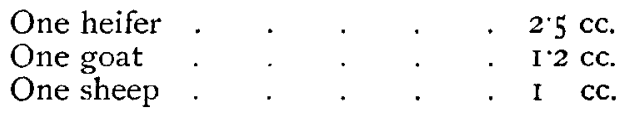

The goat died from an intervening disease. The heifer having grown very thin, it was killed at the end of sixty-seven days. The sheep offered a greater resistance to infection; it was killed on the sixty-fifth day.

In the lungs of the heifer there were granulations and small tuberculous masses, of the size of a lentil or small pea, and without an inflammatory zone. The centres showed a tendency to degeneration. The bronchial and mediastinal glands were infiltrated with a yellowish tuberculous matter. The lungs and the glands of the sheep presented similar lesions. The goat, having died prematurely, presented no very evident lesions. Nevertheless, very small sub-pleural swellings and a few fine granulations were observed in the pulmonary parenchyma.

(g) General Examination of the Author's own Experiments:-The inoculations described in the preceding paragraphs are forty-six in number-thirty-three were made with human bacilli, and thirteen were made with animal bacilli.

The human bacilli were taken from five different sources, the animal bacilli from four sources-three from the ox, one from the horse. The thirty-three animals devoted to inoculation with human bacilli were taken from the different species which provided subjects for the experiments carried out by Koch and Schuitz. It was the same with the thirteen animals used for inoculation with bacilli of animal origin.

The results were positive in every experiment.

The results of the experiments relating to inoculation with bovine tuberculosis will surprise no one. But Koch and Schiitz, as well as the partisans of their doctrine of dualism, will perhaps be astonished at the success of the inoculations made with the human bacilli. 'It is therefore well to insist on this point.

The five bacilli of human origin which we have studied have all infected the ox, sheep, goat, donkey, and pig by intravenous injection, and caused more or less extensive tuberculous lesions in all the parenchymatous viscera.

However, we must add that the virulence, as measured by pathogenic 
aptitude and toxic properties, was not the same in all. Three of them did not cause fatal tuberculosis in any of the animals submitted to the experiment, and two of the latter only showed microscopic lesions in certain species, namely the bovine species. But, thanks to histological examinations, and to the presence of subjects of several species in the series, one may be assured that the two bacilli were not entirely lacking in pathogenic power.

However, if there are human bacilli which are only slightly pathogenic for animals, there are others which are very active and of which the pathogenic power may be compared with that of the majority of bacilli of bovine origin. Thus, one of ours, that of the second series, caused fatal tuberculosis in the ox and sheep; another, that of the third series, killed the ox, sheep, and goat, just like the bacilli of bovine origin, and in as short a lapse of time.

We must add, to be complete, that, as a general rule, bovine tuberculosis has shown itself to be less infective. Whereas almost all the subjects inoculated with bovine tuberculosis died from the infection, and not later than forty-four days afterwards, the majority of those inoculated with human bacilli had to be killed. But the subjects killed by the infection were not scattered about in the different series; they belonged to particular series, from which we must conclude that among the bacilli from different sources some are very virulent.

It is therefore impossible for us, after our experiments, to accept the criterion laid down by Koch and Schiitz for separating human tuberculosis from bovine. On the question of the duality of tuberculosis, we therefore entirely disagree with the German savants. ${ }^{1}$

\section{CRITICISM OF THE VALUE OF NEGATIVE AND OF POSITIVE RESULTS, INTERPRETATION OF RESULTS.}

Before finishing, our duty is to look for the cause of the divergencies in the results obtained by Koch and Schutz and those obtained by the majority of the other experimenters who have studied this question.

We admit that Koch and Schiitz may have failed to transmit human tuberculosis to large herbivorous animals, although we make reservations with regard to several experiments which they judged as negative, either because they employed an entirely personal method of estimation, or because they did not look for lesions in microscopic sections and in all the parenchymatous viscera. ${ }^{2}$ But these negative results, if they existed, can avail nothing against the positive results obtained on all hands. Nevertheless, in order to work scientifically, we must look for the determining factor, and see if it cannot be reconciled with the dogma of the identity of human and bovine tuberculosis.

To us, the negative, or quasi-negative, results cenote the feeble virulence of the bacilli used in inoculation; while the more or less serious positive results, sometimes equal to those following an inoculation with bovine tuberculosis, are due to bacilli which are more virulent.

In our article which appeared in the Revue de la Tuberculose for

1 Immediately after Koch's lecture at the Congress in London, Lister, Nocard, M'Fadyean, Thomassen, and Ravenel expressed disagreement with the lecturer, or made strong reservations with regard to his position.

" We dealt with this point in our communication to the "Académie de Médicine," on the 24th Dec. 1901. 
August I90I, we said on this subject: "I explain the pathogenic differences observed in the experiments of Koch and Schitz by the variations in the virulence of the bacillus, and by the particular degree of susceptibility possessed by certain species of animals, and even by individuals."

And in December of the same year, in a communication to the Academie de Médicine, we said: "As for the tuberculous virus, Koch and Schütz reason as if it had a fixed and invariable activity. Koch admitted this fixity in his first works on tuberculosis. Since then it has been shown that the bacillus of tuberculosis, like many other microbes, presents frequent variations in vivo and in vitro, and I consider it an honour to have contributed to the establishment of this opinion, either directly or through some of my pupils," several of whom are now teachers.

In short, we believe we were perhaps the first in I 884 and I 886 to fix attention on the attenuation of the tuberculous virus, on the occasion of an experimental study of the tuberculous lesions called scrofulous, and of visceral tuberculosis. ${ }^{1}$

The differences which we have pointed out between the forms of tuberculosis have been recognised as exact ; but the explanation which we gave has not been readily accepted. Instead of allowing, as we do, that it depends on the difference of the activity of the virus, Eve, Straus, de Renzi, Leloir, and Nocard preferred to put it down to the number of bacilli present in the lesions. ${ }^{2}$ At first these authors did not believe, and some never have believed, in a real attenuation of the bacilli.

In spite of the objections which have been raised, we have continued our comparative studies, which embraced the diverse manifestations of surgical tuberculosis (tuberculosis of bones and joints). Among these forms of surgical tuberculosis we have found the tuberculous virus with several degrees of activity, using as test the susceptibility of the rabbit and the guinea-pig. ${ }^{3}$

A little later, in conjunction with Courmont, we examined lupus, and we proved that the virus contained in the lesions of cutaneous tuberculosis designated by this name was far from being always of the same activity. ${ }^{4}$ Finally, at my instigation, Courmont and Denis studied the degree of virulence in several cases of pulmonary tuberculosis of which the clinical symptoms were different, and there again we were able to observe that certain cases were associated with very virulent bacilli, and others with attenuated bacilli. ${ }^{5}$

We have published some experiments with the object of showing that the weakened pathogenic properties of certain tubercular viruses depend more on the attenuation than on the diminution of the number of the bacilli. We have gathered together all the facts in our Leçons sur la tuberculose ${ }^{6}$ pp. 46 and following. Nevertheless, we have not yet succeeded in convincing Leloir, Straus, and Nocard, as is easily seen from what took place at the Congress for the study of tuberculosis held at Paris in I 892, and in reading Straus's book, La tuberculose et son bacille, pp. 788 and following.

1 See Arloing: "Compte rendu de l'Académie des Sciences," 1884 and 1886. Also "Revue de Médicine," 1887.

2 See Congress for the study of Tuberculosis, 1886, p. 41.

3 See Congress for the study of Tuberculosis, 1899, p. 404.

4 See Arloing and Courmont : Congress de la Tuberculose, 1893.

"See Courmont and Denis : "Revue de la Tuberculose," 1894.

6 See Arloing : "Leçons sur la Tuberculose," Paris 1892. 
From our earlier studies we were therefore prepared to take an interest in the conflict raised by Koch, and to attempt to solve it by the variability of the bacillus of tuberculosis. The variability of the bacillus in its manner of vegetation, in its form, in his virulence, exists in bovine tuberculosis quite as well as in human tuberculosis, with this restriction, which it seems prudent to make at present, that the most attenuated varieties of the bovine bacillus have a higher virulence than the average human bacilli, So that we imagine the bacilli of human and animal origin as forming a scale of virulence, with numerous and undetermined limits. Now, these varieties were formed in some way unknown to us, by the action of living and inert media, and some are adapted so exactly to certain organisms that they seem to have changed their properties when they are suddenly transferred to other species of animals. But prolonged observations in the domain of tuberculosis permitted of the discovery of the phylogeny of these varieties, which no one would be inclined to regard as different species.

To come back to the question which we had put to ourselves, we will say that Koch and Schütz have experimented with two varieties, one human, the other bovine, as different as possible from one another in virulence. Hence the results, rather superficially gathered together, which caused them to admit a fundamental difference between the two varieties. If they had tried several bacilli of different origin, they would probably have met with intervening virulences which would have prevented them from digging a veritable trench between human and bovine tuberculosis.

In that respect, our experiments differ from theirs, and offer a peculiar interest.

The explanation which we offer to-day, renewed from that of $190 \mathrm{I}$ and my communication made last year at the Conference in Berlin, with a view to conciliation, is now generally accepted, except by Koch.

A great variation in the virulence of the bacillus of tuberculosis is readily admitted. This proves that the question has advanced since I 892, at which date we were not agreed to profess belief in the variability of Koch's bacillus. We have therefore felt great satisfaction in seeing our opinion confirmed by the works of Th. Smith, Dinwiddie, de Jong, Ravenel, Vœgedes, Veszpremy, von Behring and his pupils, and by the communications of our learned colleague $M$. Nocard, whom we formerly regretted to see amongst our adversaries. And we begin to hope that when Koch and Schütz shall be pleased to experiment under the inspiration of this opinion, these divergences will cease.

\section{CONCLUSIONS.}

From the facts contained in the above article we draw the following conclusions :-

I. Human tuberculosis can be readily inoculated into the ox, and sometimes and by certain ways of inoculation, it causes the lesions characteristic of bovine tuberculosis.

2. The human bacillus is not always of the same virulence, and a given bacillus does not manifest the same degree of activity on herbivorous animals of different species.

3. In some cases, the human bacillus is as virulent as a bacillus of 
bovine origin; in others, on the contrary, its virulence is so much weakened as to appear null, particularly if used on bovine subjects.

4. Human bacilli of attenuated virulence always cause, at least in the lung, after intravenious injections, lesions visible to the microscope, which sometimes tend to rapid fibrous transformation.

5. It is therefore impossible to give an opinion on the failure of an inoculation without a microscopic study of the lung and of the principal parenchymatous viscera.

6. The variability of the virulence of the bacillus accounts for the apparently negative results which led Koch and Schuitz to believe in duality.

7. The identity of human and bovine tuberculosis ought to be maintained, and the prophylactic measures which result from it ought also to be maintained, notably with regard to the use of milk.

\section{INTUSSUSCEPTIONS}

By Edredd M. Corner, B.Sc. (London), M.A., M.B., B.C. (Cambridge), F.R.C.S. (England), Demonstrator of Anatomy, late Resident Assistant Surgeon, St. Thomas' Hospital.

DURING the years I9OI and I9O2 a number of cases of intussusception passed through my hands, and from observations that were made upon them I became convinced that the present state of our knowledge of the pathology of this condition was erroneous. In a paper in the St. Thomas' Hospital Reports, I9OI, I raised this question, embodying also my reasons for the attitude taken up and the reports of five clinically interesting cases. If the histories of the sister sciences of anatomy, physiology, etc, are examined, the fact at once becomes patent that although the knowledge gained from mere dissection was continually increasing, no advance of importance was made until the introduction of the comparative method. This comparative method has been used in physiology, medicine, and pathology, and to a less extent in surgery. But observers are much hampered here by reason of their lack of knowledge of the physiological limits of the animals experimented on. Veterinary surgery and medicine have only of recent years been pursued with true scientific spirit, and in this respect are still far behind their corresponding human sciences. As a result, they are as yet scarcely ripe for accurate comparisons ; yet, on account of the larger size of the parts that may be observed, and the still greater frequency of post-mortem observations, a great deal may be learned.

In $1898 \mathrm{I}$ made use of this method in an enquiry into the influence of the quadrupedal and bipedal positions on fractures and dislocations in man and animals. ${ }^{1}$ The interest of such a comparison has caused me to examine the records of intussusception in animals to see how they shed light upon the pathology. The knowledge gained from this enquiry forms the subject matter of this paper, and I have added a short introductory account of the present views on the pathology of intussusceptions and the alterations that I have suggested.

Before proceeding, I would like to offer my thanks to Prof. Hobday for his scientific interest in my work and the readiness with which he placed his knowledge at my disposal.

$$
1 \text { "Lancet," } 1898 \text { and 1899, "Veterinarian," } 1899 .
$$

